
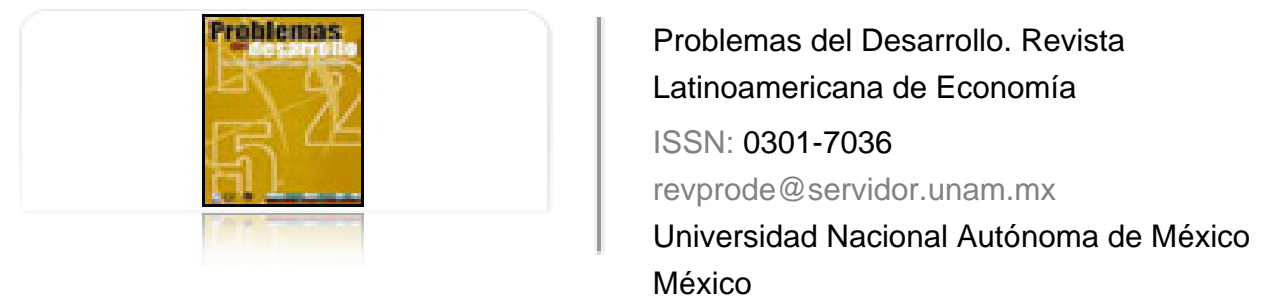

Escalante Semerena, Roberto I.; Lugo Olmos, Igor Relación entre el crecimiento económico y las economías externas de aglomeración en México Problemas del Desarrollo. Revista Latinoamericana de Economía, vol. 36, núm. 141, abril-junio, 2005, pp. 131-153

Universidad Nacional Autónoma de México

Distrito Federal, México

Disponible en: http://www.redalyc.org/articulo.oa?id=11820075008

- Cómo citar el artículo

- Número completo

- Más información del artículo

Página de la revista en redalyc.org

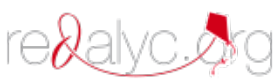

Sistema de Información Científica

Red de Revistas Científicas de América Latina, el Caribe, España y Portugal Proyecto académico sin fines de lucro, desarrollado bajo la iniciativa de acceso abierto 


\title{
RELACIÓN ENTRE EL CRECIMIENTO ECONÓMICO Y LAS ECONOMÍAs EXTERNAS de AGLOMERACIÓN EN MÉXICO
}

\section{Roberto I. Escalante Semerena* Igor Lugo Olmos**}

Fecha de recepción: 15 de octubre de 2004. Fecha de aceptación: 13 de mayo de 2005.

\section{Resumen}

Se intenta explicar un comportamiento nacional de rendimientos crecientes en la escala productiva. El objetivo del trabajo consiste en estudiar y medir el impacto de la urbanización en el crecimiento económico de país. Se usaron, como principales líneas teóricas, las aportaciones del crecimiento endógeno, al cual, además de los principales factores de producción, capital y trabajo, se añade el elemento de externalidad. La principal aportación científica del texto se localiza en los estudios de economías de aglomeración, que explican la naturaleza y medición de las externalidades por medio de diferentes técnicas econométricas. Los datos usados en series de tiempo, para el caso de México, proporcionan una opción metodológica para realizar análisis de externalidades de forma agregada que no se habían llevado a cabo en las comunidades económicas nacional e internacional.

Palabras clave: economías de aglomeración, urbanización, concentración urbana, índice Hirschman-Herfindahl, crecimiento económico, cointegración, exogeneidad, modelo de corrección de errores.

\begin{abstract}
The aim here is to explain the movement of growing national yields to scale of production. The objective of the study involves studying and measuring the impact of urbanization on the country's economic growth. As a major theoretical line the contributions of endogenous growth were used, where the element of externality is added to the main factors of production, capital and labor. The principal scientific contribution of this work centers on the study of cluster economies, which account for the nature and measurement of externalities by means of various econometric techniques. The data used in the time-series, in the case of Mexico, provide a methodological option for carrying out an analysis of externalities in aggregate form which had not been done in the national and international economic communities.
\end{abstract}

Key terms: cluster economies, urbanization, urban concentration, Hirschman-Herfindahl index, economic growth, co-integration, exogeneity, error correction model.

* Director de la Facultad de Economía, FE-UNAM. Correo electrónico: semerena@servidor.unam.mx

** Doctorante, FE UNAM. Correo electrónicolugoigo@yahoo.com 


\section{Résumé}

On essaye d'expliquer un comportement national de rendements croissants à échelle dans la production. L'objectif de ce travail consiste à étudier et à mesurer l'impact de l'urbanisation dans la croissance économique du pays. On a utilisé, comme principale ligne théorique, les apports de la croissance endogène, dans laquelle, en plus des principaux facteurs de production, capital et travail, on ajoute l'élément d'externalité. L'apport scientifique principal de cette étude se trouve dans les études des économies d'agglomération, qui expliquent la nature et la mesure des externalités au moyen de différentes techniques économétriques. Les données utilisées dans des séries de temps, pour le cas du Mexique, offrent une option méthodologique pour la réalisation d'analyse d'externalités de forme ajoutée qui n'avaient pas été réalisées dans les communautés économiques nationale et internationale.

Mots clés: economies d'agglomération, urbanisation, concentration urbaine, indice HirschmanHerfindahl, croissance économique, cointégration, exogénéité, modèle de correction d'erreurs.

\section{Resumo}

Tenta-se explicar um comportamento nacional de rendimentos crescentes a escala na produção. O objetivo do trabalho é estudar e medir o impacto da urbanização no crescimento económico do país. Como principal linha teórica usaram-se as aportações de crescimento endógeno, no qual, além dos principias fatores de produção, capital e trabalho, adiciona-se o elemento da externalidade. A principal aportação científica do estudo encontra-se nos estudos de economias de aglomeração, que explicam a natureza e medição das externalidades por médio de diferentes técnicas econométricas. Os dados usados em séries de tempo, para o caso do México, fornecem uma opção metodológica para realizar análise de externalidades de forma agregada que não se tinham efetuado nas comunidades económicas nacional e internacional.

Palavras chave: economias de aglomeração, urbanização, concentração urbana, índice Hirschman-Herfindahl, crescimento económico, cointegração, exogeneidade, modelo de correção de erros. 


\section{Introducción}

$\mathrm{E}$

1 análisis económico en materia de crecimiento ha incorporado la urbanización rural —con instituciones y políticas económicas informales — a uno urbano — con instituciones formales y anonimato relativo- se conoce como urbanización (Henderson, 2003b), fenómeno multidimensional que se refleja en el orden básico de la sociedad, en los aspectos económico, político, social, psicológico y demográfico-ecológico (Unikel, 1972).

Estudios empíricos realizados por Fay y Opal (2000) y por Davis y Henderson (2003) mencionan que la política gubernamental es el punto clave que afecta la urbanización, mediante modificaciones en la composición de los sectores. Una de las hipótesis planteadas por Gallup, Sacks y Mellinger (1999) la señala como causa del crecimiento económico; no obstante, la evidencia econométrica encontrada por Henderson (2003a) y Lugo (2004) indica una falta de relación causal entre las dos variables. Por lo tanto, la hipótesis se reestructura con el planteamiento de que la urbanización promueve la formación de aglomeraciones económicas cuyos efectos se pueden apreciar en el crecimiento económico (Henderson, 2003b). Al especificar el problema, se obtienen las preguntas clave: ¿por qué la urbanización se concentra o se dispersa en las ciudades? y ¿cómo la concentración o dispersión urbana afecta el comportamiento del crecimiento económico?

Los tratados de economías de aglomeración, para argumentar su razonamiento, delimitan las fuerzas que provocan la caída de los costos de la actividad económica provenientes de empresas o actividades locales (Pearce, 1992). Las economías internas (de escala y especialización) o las externas (de urbanización) son los impulsos generadores de un incremento de la escala de producción (Marshall, 1890; Rosenthal y Strange, 2003). Así, los rendimientos crecientes a escala se fundamentan en la presencia de externalidades y propician la justificación teórica para relacionar el crecimiento económico endógeno con las economías de aglomeración.

Desde un enfoque de aglomeración, las externalidades se asimilan en tres ámbitos: industrial, geográfico y temporal (Rosenthal y Strange, 2003). Cada uno de ellos representa una elección metodológica para apropiarse del conocimiento referido a la naturaleza y las implicaciones de los procesos relacionados con externalidades (Arellano, 2005).

Se tomó la iniciativa de combinar los ámbitos industrial y temporal, con las fuerzas de economías externas para aproximar y medir las externalidades que provocan un crecimiento endógeno. Como el estudio pretende demostrar, de manera agregada, la relación

\section{Desaarrollo}


de factores externos con el crecimiento económico nacional, se optó por seleccionar dichos ámbitos, además de elegir una base de datos en series de tiempo.

La metodología econométrica, según Wheaton y Shishido (1981) y Henderson (2003a, 2003b), ${ }^{1}$ consiste en encontrar un vector de cointegración por medio del procedimiento de Johansen (1988), realizar pruebas de raíces unitarias, construir un modelo de vectores de corrección de errores (VECM) para poder realizar las pruebas de exogeneidad: débil y no causalidad de Granger y, por último, formular un VECM que señale los efectos dinámicos de las economías externas. El índice Hirschman-Herfindahl apoya el análisis para medir la influencia de externalidades que afectan el crecimiento económico nacional.

El estudio se hizo con datos del Sistema Urbano Nacional del Consejo Nacional de Población (CONAPO), de los censos de población y vivienda y del Banco de Información Económica del Instituto Nacional de Estadística, Geografía e Informática (INEGI).

Según los principales resultados, se observa un proceso de urbanización caracterizado por la dispersión urbana, la cual influye en el crecimiento económico nacional. A partir de la influencia de las economías externas que generan externalidades estáticas y dinámicas, se puede identificar como endóngeno el crecimiento del periodo 1970-2001.

En el primer apartado se especifica la base teórica con la cual se intentará relacionar la teoría del crecimiento endógeno con la urbanización. En el segundo se analizarán esta última y sus formas de expresión. En el tercero se tratan los modelos teórico y econométrico, relacionando la teoría del crecimiento endógeno con el índice $\mathrm{H}$. El cuarto muestra la estructura y manejo de datos. Y, por último, se señalan los resultados empíricos para México, así como las conclusiones.

\section{La teoría}

La teoría económica del crecimiento, que es la base del presente análisis, sigue la tradición neoclásica y, de manera particular, se enfoca en los modelos de crecimiento endógeno y en los aportes de Arrow (1962), Romer (1986) y Lucas (1988), en los cuales se argumenta que el crecimiento económico en el largo plazo puede asociarse con el comportamiento de la inversión o acervo total de capitales, el trabajo y la tecnología usada, además de que presenta efectos de escala causados por externalidades. Los resultados de esta formulación teórica no dependen de la especificación de un cambio tecnológico exógeno o de diferentes dotaciones iniciales entre países; las preferencias y la tecnología son idénticas y estacionarias.

Considerar el cambio tecnológico endógeno dentro de un modelo de equilibrio determina que la acumulación de conocimiento guíe el crecimiento económico en el largo plazo (Romer, 1986). Las externalidades surgidas por la inversión en conocimiento se conside-

Modelos de corte transversal y de panel.

\section{Desarrrollo}


ran naturales y, por lo tanto, este último genera una productividad marginal creciente. Arrow (1962) señala que al ser adquirido por las empresas se vincula con la experiencia y desarrolla una estrecha relación entre ésta y los aumentos en la productividad. Así, se obtienen rendimientos crecientes cuando un nuevo conocimiento se descubre, se invierte en él y se aplica a la producción (Arrow, 1962; Romer, 1986). En la formalización de un modelo de optimación del crecimiento en presencia de rendimientos crecientes, autores como Arrow (1962), Levhari (1966a, 1966b) y Sheshinski (1967) demostraron que, en el agregado, la tasa de crecimiento del producto per capita es una función monótona creciente de la tasa de crecimiento de la población, ${ }^{2}$ lo cual se conoce como efectos de escala. La unidad de referencia al hablar de estos efectos es el espacio, relacionado con un área en la cual se desborda determinado tipo de conocimientos. Dichos efectos explican una relación similar entre crecimiento de la población y movimientos del producto a lo largo del tiempo, sin llevar a un estado estacionario, y el aumento en la tasa de crecimiento económico.

Las externalidades de escala se consideran el punto de unión entre los aportes teóricos del crecimiento endógeno y la urbanización (Black y Henderson, 1999), las cuales ya han sido objeto de investigación (Combes, 2000; Ciccone y Hall, 1996; Henderson, 1997; Henderson, Kuncoro y Turner, 1995; Gleaser, Khalil, Scheinkmann y Schleifer, 1992). El núcleo teórico de inicio lo estableció Marshall (1890), al explicar que las externalidades son resultado de un derrame de información local. ${ }^{3}$ El análisis se refiere a empresas establecidas en una localidad que aprenden de aquellas establecidas en su misma industria. Desde este supuesto, las externalidades de escala estáticas son llamadas de localización o, en su forma dinámica, externalidades Marshall-Arrow-Romer (MAR). Esto explica la existencia de una o varias actividades fuertemente relacionadas entre sí en una pequeña área de influencia. De forma alternativa, si se da ese aprendizaje en el mismo sitio, con la premisa de una diversidad industrial, las externalidades estáticas son llamadas de urbanización o, en un contexto dinámico, externalidades de Jacobs (1969). El crecimiento de una industria se sostiene en la diversidad de actividades y en un amplio ambiente urbano; ambas formas de externalidades implican una relación con el desarrollo en este ámbito (Jacobs, 1969; Henderson, Lee y Lee, 2001). Las externalidades dinámicas señalan la importancia actual del comportamiento industrial histórico de las ciudades, presentando un stock de conocimientos acumulados en el tiempo y reduciendo la movilidad industrial (Romer, 1986; Lucas, 1988; Rauch, 1993).

2 En la formalización más desarrollada de los libros de texto, esta idea se expresa con mayor claridad por medio de la tasa de crecimiento del capital per capita y su relación con el tamaño de la población como en Sala $\sim \mathrm{i} \sim \operatorname{Martin}(1994): \gamma_{k}=s A L^{\eta}-\delta$, donde $\mathrm{S}=$ tasa de ahorro, $\mathrm{A}=$ progreso técnico, $\mathrm{L}=$ trabajo, $\delta=$ depreciación y $\eta=$ importancia de las externalidades.

3 Se hace referencia a un mercado local de recursos, productos y tecnologías específicas.

\section{Desaarrollo}




\section{La urbanización y sus formas}

Al parecer, el crecimiento económico y la urbanización tienen una estrecha relación. La concordancia entre ambos debe fundamentarse por medio de la influencia de la urbanización sobre la formación de economías externas de aglomeración. Este fenómeno se caracteriza por generar importantes cambios en la distribución y composición de la población, e incorporar progresivamente grandes sectores sociales a un estilo de vida urbano, es decir, por transformaciones continuas y aceleradas en la estructura rural-urbana (González, 2001). Con el argumento de que la urbanización afecta directamente la distribución espacial de la población y las actividades económicas, es fundamental relacionar el factor regional con las modificaciones en la composición de sectores económicos (De Pablo, 1983).

Generalmente, el desarrollo económico de los países en vías de desarrollo está acompañado por un crecimiento urbano explosivo, el cual depende de tres factores: la migración rural-urbana, la jurisdicción administrativa y el incremento natural de la población. El primero se debe a una racionalidad económica fundamental, mientras que los otros tienen que ver con el factor político de desarrollo rural (Bruekner, 1990; Kojima, 1996). La decisión de los individuos de migrar hacia otras zonas es una respuesta a los incentivos —dentro de ellos sobresalen los salarios-y oportunidades económicas características de la urbanización, independientemente de que estas áreas se encuentren en un esquema de empleo débil (Fay y Opal, 2000).

Existen tres dificultades que impiden respuestas significativas en la relación crecimiento económico-urbanización (Henderson, 2003a). Primera: la urbanización no es una fuerza que promueve el crecimiento per se, sino el resultado de una variedad de factores relacionados con el cambio en la composición del producto nacional y las condiciones sociales, es decir, en esta primera observación se le considera una variable exógena (Lugo, 2004). Segunda: la urbanización es un proceso transitorio, en el cual, si existiera crecimiento económico, en algún momento, todos los países tendrían una total urbanización. Finalmente, su definición varía dependiendo de qué es considerado urbano en cada país.

Se dice que la forma de $S$ que presenta la correlación entre porcentaje de urbanización y producto per capita es una relación informal, porque el primero sigue el mismo modelo que el crecimiento de la población, en otras palabras, la transición demográfica entre la tasa de mortalidad y la de fertilidad (Henderson, 2003b). El crecimiento de la población es menor en bajos niveles de crecimiento económico; acelera su ritmo de desarrollo, seguido de una disminución con un aumento en el producto por trabajador. La hipótesis manejada (Henderson, 2003a) es que, a medida que el ingreso per capita aumenta, existe un aumento en el grado de urbanización. Al considerarla como factor que promueve el crecimiento, se puede caer en la falsa idea de que países con bajos niveles de ingreso y altos grados de agricultura de subsistencia y de analfabetismo puedan presentar una completa urbanización. Así, los beneficios intrínsecos de la urbanización son promover for- 
mas de aglomeración en las cuales se presente una difusión de conocimientos e información que promueva el crecimiento económico (Henderson, 2003a, 2003b). Esta última afirmación explica que fuerzas de aglomeración externas, resultantes de la urbanización, faciliten los efectos de escala.

Las fuerzas de concentración o dispersión provienen de las economías de aglomeración. Las economías de urbanización reúnen o derraman recursos y actividades en una o varias ciudades (Henderson, 2003a). La hipótesis de Williamson (1965), adoptada en un contexto urbano por Hansen (1990), expone que, en un inicio, el mejor punto de concentración aumenta con un ingreso bajo, para después alcanzar el más alto grado de concentración con un mayor nivel de ingreso, y empieza a caer la concentración con mayores aumentos en el ingreso. Como ejemplo, se señala que una concentración industrial espacial localizada en ciudades conserva la infraestructura económica - física de capital: telecomunicaciones y transporte- y el manejo de recursos, lo cual permite un flujo de información y conocimientos. Ante un proceso de desarrollo creciente, eventualmente se presenta una dispersión de actividades por dos razones: a) la economía extiende sus recursos de infraestructura y conocimiento hacia áreas interiores, y b) la concentración inicial en las ciudades empieza a tener altos costos. Se llega a un punto de saturación en el cual disminuye la eficiencia espacial para los productores y los consumidores (Henderson, 2003a; Davis y Henderson, 2003). Varios autores (El-Shakhs, 1972; Alonso, 1980; Wheaton y Shishido, 1981; Junius, 1999) han propuesto un modelo de crecimiento mediante la relación entre disminución de concentración urbana y aumento del ingreso per capita.

\section{Concentración y dispersión urbana}

Para formalizar las mediciones relacionadas con economías de aglomeración y externalidades, existen dos propuesta en la literatura (Wheaton y Shishido, 1981; Jefferson, 1939; Mutlu, 1989; Henderson, 2003a y 2003b) que intentan aproximar el grado de concentración o dispersión urbana. La primacía define, inicialmente — en el sentido de Jefferson-, que el tamaño de la ciudad principal es desproporcionadamente mayor en relación con el de una segunda. Cualquiera que sea el tamaño de la primera con referencia a la segunda, si aquella excede del doble, se dice que tiene primacía (Jefferson, 1939; Mutlu, 1989). La segunda medición usada es el índice Hirschman-Herfindahl $(H)$, que señala el grado de concentración o dispersión urbana (Henderson, 2003b). La base de construcción del índice se debe a la evidencia presentada por la ley de Zipf (Gabaix, 1999; Fujita, Krugman, y Venables, 2001): cuando la jerarquía inicia de la ciudad más grande a la más pequeña, el producto de la clasificación y el tamaño de la población deben aproximarse a una misma constante para todas las ciudades. Si se presenta esa condición, el tamaño de la ciudad mayor delinea la dimensión de las restantes, información suficiente para calcular cualquier índice comparativo de concentración urbana (Henderson, 2003a; Davis y Henderson,

\section{Desaarrollo}


2003). Si $R$ se detalla como una lista jerarquizada de ciudades y con $n$ se considera el tamaño de la ciudad, la relación se expresa:

$$
R(n)=A n^{-\alpha}
$$

donde la $\operatorname{Prob}(\operatorname{tamaño}>n)=A n^{-\alpha}$. Con la ley de Zipf, $\alpha=1$ y $A$ es una constante determinada por la jerarquía (Henderson, 2003b). Si $\alpha$ se acerca a la unidad, se aproxima al cumplimento de dicha ley.

Sobre la base de ésta, el índice Hirschman-Herfindahl se calcula por medio de la siguiente fórmula:

$$
H=\sum_{i=1}^{n}\left(\frac{P_{i}}{P}\right)^{2}
$$

en donde $P_{i}$ es la población de la ciudad $i, P$ es la población urbana total y $n$ es el número de ciudades incluidas en el procesamiento de datos. El inverso del índice, $1 / H$, se puede considerar un índice de dispersión que varía de 1 - en caso de concentración total一 a 0 en casos de total dispersión (Wheaton y Shishido, 1981). Para mantener la uniformidad teórica y empírica del análisis, se hablará del inverso del índice $H$ como la principal medida que describe la dispersión urbana.

En la formación técnica del índice se presentan dos importantes cuestiones por decidir: ¿qué unidades de observación se usarán? y ¿ciudades o áreas metropolitanas? Si se opta por el tamaño de las ciudades, el análisis se basa en los límites políticos definidos para cada una de ellas. En su definición, las zonas metropolitanas incluyen a toda la población que vive dentro de un ámbito urbano económicamente cohesivo, es decir, es un área en donde cada componente se interrelaciona funcionalmente con otras partes de la misma, usualmente mediante el comercio y sus comunidades (Mera, 1973; Wheaton y Shishido, 1981). Si se eligen las áreas metropolitanas como unidades de observación, es indispensable usar algún tipo de criterio para limitar y agrupar su tamaño. Una opción es eliminar aquellas áreas que tienen un efecto mínimo por su dimensión, sin embargo, tiene la desventaja de recargar las diferencias en el número de áreas utilizadas en cada país; por lo general, los países en vías de desarrollo limitan su estudio a una o dos. El criterio alternativo, que necesita un tratamiento más complejo, es utilizar las zonas metropolitanas más grandes que representen una fracción del total de la población urbana dentro de un rango de 65\% a 70\% (Wheaton y Shishido,1981; Mutlu, 1989).

A diferencia de las ciudades, las áreas metropolitanas presentan la mayor expresión de la herencia del proceso de urbanización, ya que reúnen los beneficios presentados por 
externalidaes de escala locales y las actividades congregadas en sus áreas periféricas (Henderson, 1988, 2003b). Estas características las hacen centros de innovación, de dirección y de servicios (Kolko, 1999). Reunir y cuantificar estas grandes áreas enriquece el análisis y puede representar una mejor aproximación para formar un indicador del grado de dispersión urbana (Lugo, 2004).

\section{Especificación del modelo}

Para especificar el modelo se partió de una función de producción Cobb-Douglas, neutral en el sentido de Harrod, ${ }^{4}$ de la forma:

$$
Y_{t}=K_{t}^{\alpha}\left(A_{t} L_{t}\right)^{1-\alpha}
$$

donde $Y_{t}$ es el producto, $K_{t}$ es el capital, $A_{t}$ es el progreso técnico y $L_{t}$ el empleo. Reescribiendo (3) en términos de trabajo y aplicando logaritmos:

$$
\log y_{t}=\alpha \log k_{t}+(1-\alpha) \log A_{t}
$$

La ecuación (4) señala que el producto por trabajador está en función del capital por trabajador y la tecnología. Como ésta, al momento de estimar (4), puede ser interpretada como la contribución del progreso técnico al crecimiento de la producción —en la cual se encuentran todas las demás fuentes que contribuyen al crecimiento económico, a excepción de la que hace el capital mediante su rendimiento (Romer, 2002)—, su influencia se refleja en el término de error. Diferenciando (4), se obtiene una ecuación expresada en tasas de crecimiento:

$$
\log y_{t}-\log y_{t-1}=\alpha\left(\log k_{t}-\log k_{t-1}\right)+(1-\alpha)\left(\log A_{t}-\log A_{t-1}\right)
$$

El crecimiento del producto por trabajador responde al cambio del capital por trabajador y la tecnología. Las modificaciones en la tecnología se pueden medir por medio de factores relacionados con el periodo base, como la educación de la fuerza de trabajo — habilidad de adoptar nuevas tecnologías - y consideraciones internas del país que afecten la eficiencia del crecimiento — grado de apertura o urbanización (Henderson, 2003a, Glaeser, Khalil, Scheinkman y Shleifer, 1992) — . La propuesta de investigación para trabajar con datos en series de tiempo determina la incorporación de factores que posibilitan un análisis en el agregado, así como también revela posibles errores de especificación del modelo. Por lo tanto, la urbanización (expresada como concentración-dispersión urbana) es la

\footnotetext{
4 Denominado trabajo efectivo.
}

\section{Desaarrollo}


variable aproximada que cuenta con características teóricas y técnicas sólidas en estudios de economías de aglomeración (Lugo, 2004). La influencia de la educación, el grado de apertura y demás factores en el cambio tecnológico se incorporan en el término de error. Así, se llega a la especificación del cambio tecnológico como:

$$
\log A_{t}-\log A_{t-1}=f\left[(1 / H)_{t}, \varepsilon_{t}\right]
$$

La relación en logaritmos se establece de la forma:

$$
\log A_{t}-\log A_{t-1}=\log B+\phi \log (1 / H)_{t}+\log \varepsilon_{t}
$$

donde $B>0, \phi>0 . \mathrm{B}=$ parámetro que representa modificaciones provocadas por un desplazamiento de la función; $\phi=$ externalidades causantes de un comportamiento endógeno y $\varepsilon_{t}$, factores en niveles que influyen en los cambios tecnológicos. Si encontramos la ecuación que determina $\log A_{t}$ y sustituimos en (4), la relación econométrica se especifica como:

$$
\log y_{t}=(1-\alpha) \log B+\alpha \log k_{t}+\phi(1-\alpha) \log (1 / H)_{t}+u_{t}
$$

Donde $u_{t}=\log \varepsilon_{t}+\log A_{t-1}$. La evidencia empírica de $(8)^{5}$ confirma una relación positiva entre el crecimiento del producto y la inversión, apoyados por el progreso tecnológico (Levine y Renelt, 1992; Ross, 2000). Asimismo, esta relación fortalece una dependencia positiva con la dispersión urbana, basada en el progreso tecnológico proveniente de las externalidades (MacCoskey y Kao, 1998; Henderson, 1997; Henderson y Becker, 2000, Henderson, 2000; Henderson, Lee y Joon Lee, 2001; Henderson, 2003a). Variables adicionales que podrían afectar el nivel y tasa de crecimiento del producto, así como el grado de significancia estadística y valor del coeficiente de la dispersión urbana, haciendo que el factor urbanización tenga un pequeño o nulo efecto sobre el crecimiento (Henderson, 2003a), se incorporan en $u_{t}$. Especificación determinada por medio de la variable aproximada que intenta cuantificar la importancia de la urbanización. ${ }^{6}$

Si en (8) especificamos $(1-\alpha)=\lambda$, se tiene que $\alpha+\lambda<1$, rendimientos decrecientes a escala. Mientras si $\phi>0$ las externalidades de escala son resultado de una dispersión, y si $\phi<0$ lo son de una concentración. Por lo tanto, existe una función de producción con rendimientos decrecientes, en la cual, al incorporar las externalidades de escala, se produ-

5 La ecuación refleja en buena forma la especificación teórica presentada en los libros de texto (Sala i $\sim$ Martin, 1994) como: $Y_{t}=A_{t} K_{t}^{\alpha} L_{t}^{1-\alpha} \kappa_{t}^{\eta}$, donde $\kappa_{t}^{\eta}$ representa la externalidad y $\eta$ indica su peso.

6 Por ejemplo en Henderson (2003a), se considera un índice de primacía, acorde con datos en panel. Mientras que en Lugo (2004), el índice $(1 / \mathrm{H})$ es apropiado para realizar análisis con datos en series de tiempo.

\section{Desarrrollo}


Cuadro 1

Clasificación de zonas

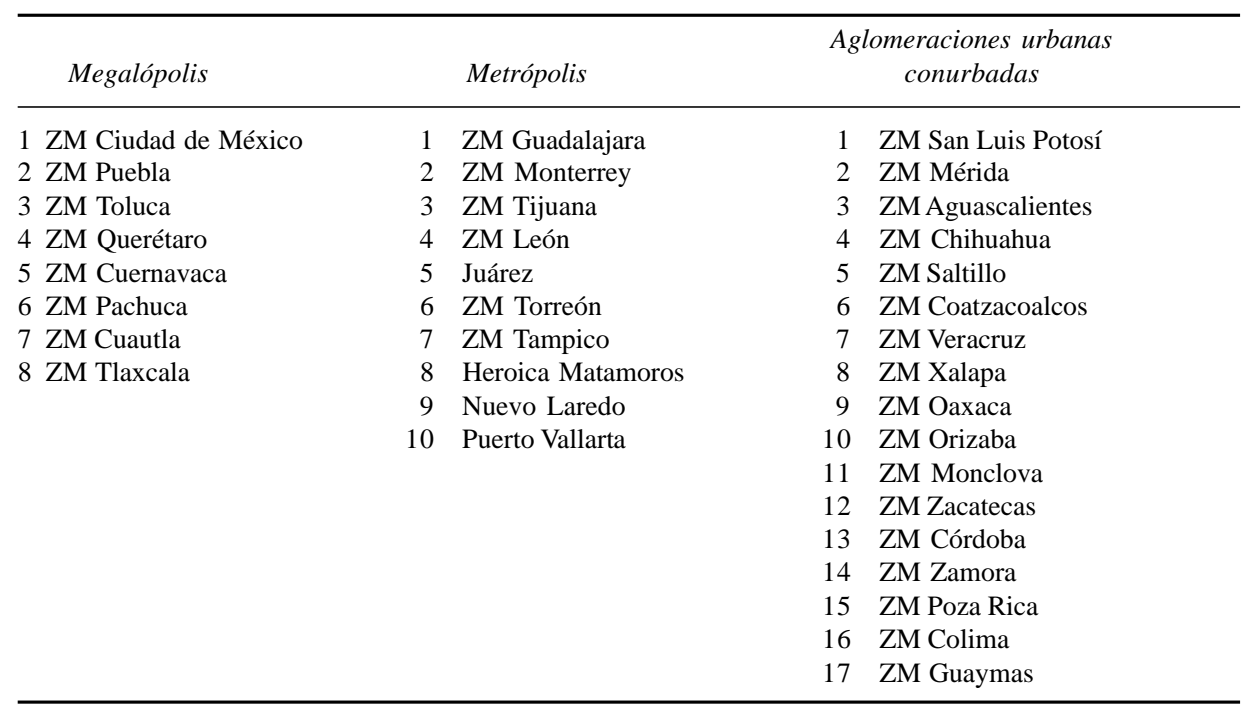

Fuente: INEGI y CONAPO.

cen rendimientos crecientes que provenien del progreso técnico, basadas en economías de aglomeración. Así, en (8) se espera que $\alpha+\phi \lambda \geq 1$.

El análisis econométrico inicia con las pruebas de raíces unitarias. Después se presenta el resultado del procedimiento de Johansen (1988) y se muestra el vector de cointegración resultante del modelo. Se realizan las pruebas de exogeneidades débil y fuerte, para concluir con un modelo de corrección de errores, reforzando la importancia de las externalidades dinámicas provenientes de economías externas de aglomeración.

\section{Datos}

Los datos cubren el periodo 1970-2001, en el cual la variable del PIB y la inversión son estadísticas anuales consultadas en el INEGI. Para representar el proceso de dispersión urbana se analizaron las principales regiones que contienen las zonas metropolitanas de la república mexicana: ${ }^{7}$ megalópolis, metrópolis y aglomeraciones urbanas conurbadas (Cuadro 1). Para formar el índice $H$ se tomó como criterio $70 \%$ del total de la población urbana como mínimo. La construcción del índice se realizó con datos del coNAPO y el INEGI (censos de población y vivienda), tomando como referencia los años 1970, 1980, 1990, 1995 y 2000. Se obtuvo un valor medio cada cinco años, calculado por promedios y tasas

7 Para jerarquizar las principales regiones se tomó como base el Sistema Urbano Nacional de 1990, con formado por 364 ciudades (31 zonas metropolitanas y 333 localidades mayores de 15 mil habitantes), identificadas con base en los datos por localidad del XII Censo General de Población y Vivienda 2000.

\section{Desaarrollo}


medias de crecimiento; después se aplicó el proceso de interpolación ${ }^{8}$ para cada zona, considerando un modelo ARIMA $(1,1,0)$, con un factor de distribución para la megalópolis de 3.19E-05, metrópolis de 1.58E-08 y las aglomeraciones urbanas conurbadas de 7.87E11. Para el total de la zona (total nacional) se consideró el mismo modelo ARIMA con un factor de distribución de 3.31E-05. Por último, se calculó el año 2001, tomando como supuesto la tasa de crecimiento del periodo anterior.

\section{Evidencia empírica}

De acuerdo con la evidencia encontrada para el caso de México, se observa una relación de $S$ entre el porcentaje de urbanización y el producto per capita como a continuación se muestra:

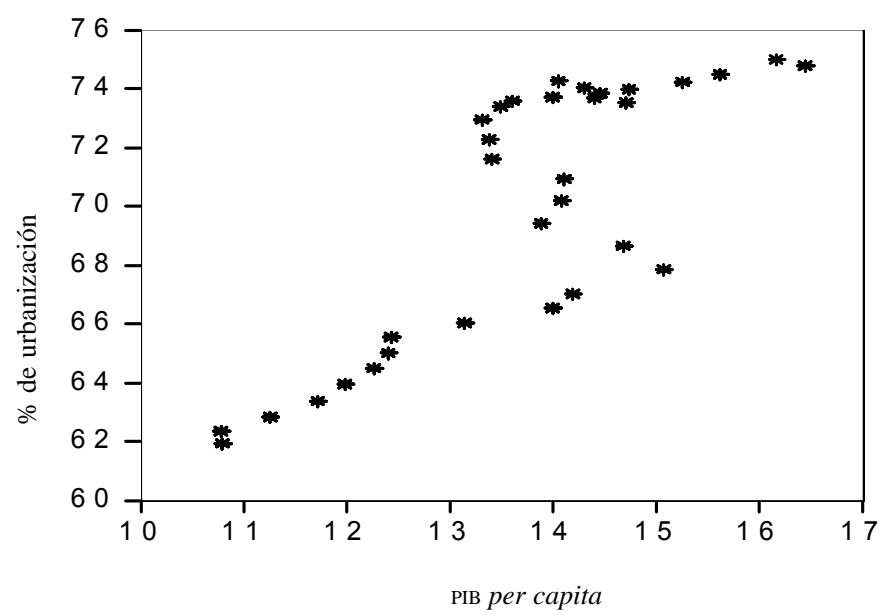

Gráfica 1. Relación entre el PIB per capita y el porcentaje de urbanización, durante el periodo 1970-2001. Fuente: Lugo, 2004.

Inicialmente se presenta un bajo nivel de urbanización, seguido de un periodo de crecimiento urbano rápido, para después estabilizar su comportamiento conforme aumenta el ingreso. Después del análisis gráfico se apoya la idea de que la urbanización influye en la conformación de aglomeraciones económicas. Además, proporciona el enlace para analizar la relación entre el PIB per capita y el índice de dispersión como se presenta a continuación.

En la Gráfica 2 se muestra la concepción de Williamson (1965), en el cual el desarrollo económico de un país se caracteriza por etapas de divergencia regional, seguidas por una fase de convergencia tardía. Es decir, algunas regiones inicialmente experimentan un acelerado crecimiento con respecto de otras (áreas periféricas), para que después éstas últimas empiecen a tener y captar mayor crecimiento.

8 Es preciso señalar que la interpolación realizada se debe considerar como proceso de submuestreo. 


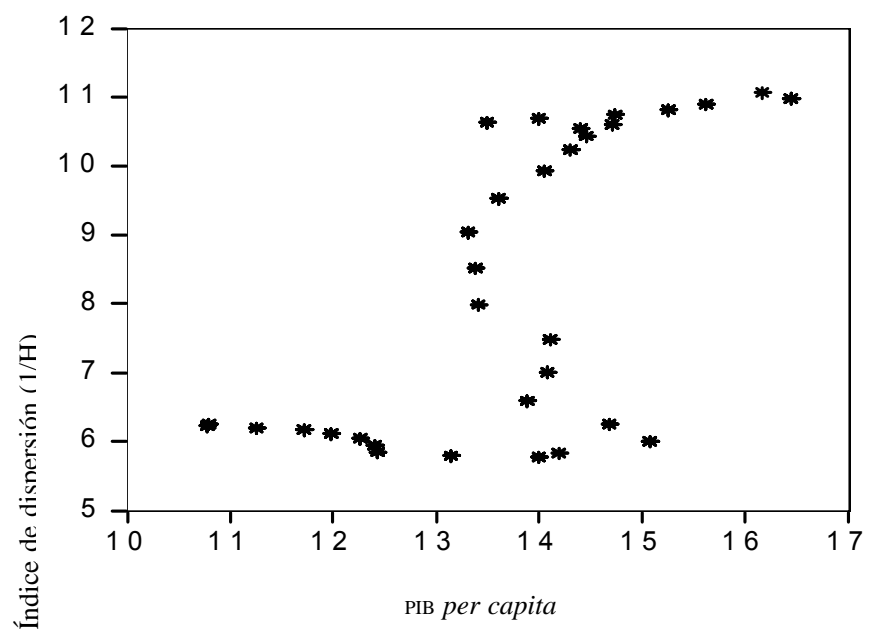

Gráfica 2. Relación entre el PIB per capita y el índice de dispersión, durante el periodo 1970-2001. Fuente: elaboración propia.

El periodo analizado se caracteriza por los cambios en el crecimiento del sistema de ciudades debido a una reconfiguración territorial y una organización del sistema urbano nacional; esto se manifesta en transformaciones en número, tamaño, función e interdependencia económica regional de las urbes que lo integran. Los cambios registrados en el total de ciudades han sido significativos. En 1900 se registraron 33; de 1940 a 1950, 55; en 1960, 123 localidades de 15 mil y más habitantes; en 1970, 166; para 1980 fueron 229; en 1990 eran 309 y al inicio del nuevo siglo se identifican 338 (Olivera, 1997; SEDESOL, 2000; González, 2001). En este periodo, el país experimentó transformaciones en el nivel internacional que han inducido a implantar nuevos modelos de desarrollo, reestructurando la economía y modificando los patrones de organización del territorio nacional. Los tratados de libre comercio con otros países han creado alternativas de desarrollo para las regiones y ciudades del país. La tecnología sustituye a la industria convencional como motor del desarrollo económico, en el cual los nuevos polos son complejos de innovación tecnológica que inciden en el funcionamiento económico de las urbes y regiones (González, 2001). Las tasas de crecimiento promedio de la población de las megalópolis disminuyen en el periodo 1980-1990; la caída es muy significativa, pasa de 5.1\% a 2.3\%. Éstas siguen también un movimiento descendente en sus tasas de crecimiento promedio, pero es una caída más uniforme. La tasa de crecimiento promedio de las aglomeraciones urbanas conurbadas de 1980 a 1990 es muy alta, lo cual confirma una transferencia de actividades económicas, sociales, políticas y culturales hacia las ciudades medias y reafirma la política de desarrollo urbana destinada a incentivar nuevos centros de desarrollo en el nivel nacional (Lugo, 2004).

\section{Desaarrollo}


A partir de los años setenta, la concentración empezaba a cambiar la dinámica del crecimiento urbano: dicha concentración disminuía, la migración campo-ciudad bajaba, al igual que las tasas de crecimiento de algunas ciudades. A finales de esta década se estableció una política urbana de descentralización de la vida nacional y el desarrollo regional. En los años ochenta esta política se orientó al equilibrio económico y social del crecimiento de las urbes, además desalentó la corriente concentradora de las zonas metropolitanas e impulsó ciudades medias (INEGI, 2000). El Plan de Desarrollo Urbano 1990-1994 tenía como objetivo principal la transformación del modelo territorial de asentamientos humanos en concordancia con políticas de descentralización y de desarrollo económico, lo cual generaría una disminución de los costos sociales de las grandes concentraciones urbanas, mejoraría la calidad de sus servicios, atendería los requerimientos básicos del suelo, infraestructura y equipamiento, y fortalecería la capacidad municipal para activar un desarrollo ordenado de las urbes. Esto impulsó proyectos estratégicos, dentro de los cuales el programa 100 Ciudades se orientó a interrelacionar desarrollo y urbanización, afectar planeación e inversión, validar la equidad económica, coordinar el federalismo y la descentralización, regular los proyectos de largo alcance, manejar la participación social y fortalecer la sustentabilidad con el desarrollo urbano (SEDESOL, 1990; González, 2001; SEDESOL-CICM, 1994).

Para representar el movimiento de dispersión urbana en el nivel nacional, se realizaron los cálculos por medio de la ley de Zipf. Se tomaron como base algunas de las principales zonas metropolitanas de México y se ordenaron de acuerdo con el tamaño de su población en el periodo 1970-2000 (véase Gráfica 3).
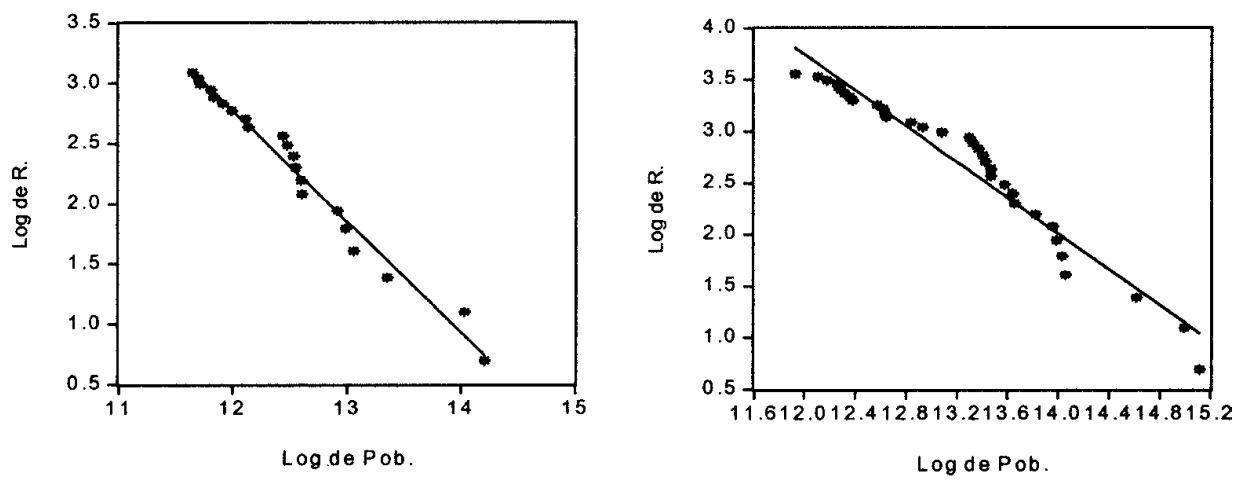

Grafica 3. Comparación del año de 1970 y 2000 por medio de la ley de Zipf.

Fuente: elaboración propia.

Se observa claramente la pendiente negativa en ambas gráficas, la cual es muy próxima a -1. Si realizamos las estimaciones para comprobar dicho comportamiento en ambos periodos se obtiene: 
RELACIÓN ENTRE EL CRECIMIENTO ECONÓMICOY LAS ECONOMÍAS EXTERNAS

\begin{tabular}{cc}
\hline Año 1970 con $22 \mathrm{ZM}$ & Año 2000 con $35 \mathrm{ZM}$ \\
\hline $\mathrm{LR}=12.17-0.78 \mathrm{LS}$ & $\mathrm{LR}=13.73-0.83 \mathrm{LS}$ \\
$(0.155) \quad(0.035)$ & $(0.140)(0.029)$ \\
\hline
\end{tabular}

Nota: El valor entre paréntesis es el error estándar. LR y LS son la jerarquía y tamaño de la ciudad en logaritmos respectivamente.

Al comparar ambos resultados, los valores de $\alpha$ se aproximan a -1, lo cual sugiere la existencia de movimientos de dispersión urbana. Dependiendo del número de ciudades considerandas en el cálculo aumenta el valor de $\alpha$. La homogeneidad en el proceso de crecimiento de las urbes está estrechamente relacionada con la ley de Gibrat y el teorema del límite central (Sutton, 1997; Gabaix, 1999; Henderson, 2003b).

El análisis en tasas de crecimiento entre el producto y el índice de dispersión muestra un comportamiento inverso. La estructura dinámica en ambas variables se ejemplifica por medio del filtro Hodrick-Prescott (véase Gráfica 4). ${ }^{9}$

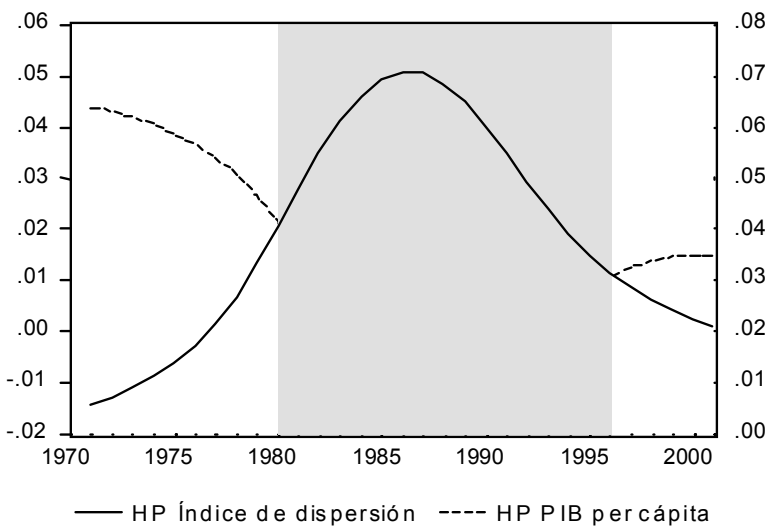

Gráfica 4. Filtro Hodrick-Prescott de la tasa de crecimiento del producto per capita y el índice de dispersión en el periodo 1970-2001.

Fuente: elaboración propia.

Cuando el producto per capita se encuentra en su tasa de crecimiento más baja, periodo 1985-1987, la dispersión urbana se localiza en su tasa máxima. El comportamiento inverso de ambas variables sugiere un cíclico, aproximadamente de 15 años, cuyo equilibrio se da en 1980 y 1996. Por lo tanto, dicha dispersión pretende influir en el crecimiento económico. Al encontrarse en un punto máximo de dispersión, las actividades de las principales zonas y ciudades empiezan un proceso de concentración caracterizado por recuperar la

9 Aunque el filtro se utiliza para analizar ciclos económicos, la exposición del mismo se realiza con el afán de esclarecer los movimientos inversos en ambas variables. 
distribución en tamaño y actividad económica regional. La existencia de un progreso de concentración urbana trae consigo etapas de crecimiento económico, mientras que una fase de dispersión disminuye el ingreso per capita. Consecuentemente, la evolución de concentración-descentralización urbana genera y equilibra las condiciones necesarias para promover un crecimiento económico nacional.

Para determinar el orden de integración de las variables se utilizaron las pruebas de raíces unitarias Dickey Fuller Aumentada [ADF, por sus siglas en inglés] (1981), PhillipsPerron, PP (1988) y Kwiatkoski, Phillips, Schmidt y Shin, KPSS (1992). Las dos primeras pruebas se especificaron incluyendo una constante, tendencia y constante, y sin constante y tendencia. La selección de la especificación utilizada en las pruebas de Dickey-Fuller y Phillips-Perron se obtuvo mediante el procedimiento de lo general a lo específico, estimando regresiones con constante y tendencia, y verificando su significancia estadística. El número de rezagos $(\rho)$ se seleccionó de acuerdo con el criterio de significancia estadística de la prueba $t$, procedimiento que es conocido como $t$-sig (Ng y Perron, 1995). Se estimó la prueba KPSs, cuya hipótesis nula plantea que la serie es estacionaria, a diferencia de las pruebas ADF y PP, en la cual la serie tiene raíces unitarias, lo que permite minimizar la

Cuadro 2

Pruebas de raíces unitarias ADF, PP, KPSS y la prueba conjunta ADF-KPSS

\begin{tabular}{|c|c|c|c|c|c|c|c|c|c|c|}
\hline \multirow[t]{2}{*}{ Variable } & \multicolumn{3}{|c|}{$A D F$} & \multicolumn{3}{|c|}{$P P$} & \multicolumn{2}{|c|}{ KPSS } & \multicolumn{2}{|c|}{$\begin{array}{c}\text { Prueba conj } \\
\begin{array}{c}\text { ADF-KPSS } \\
\rho=0.75\end{array}\end{array}$} \\
\hline & $A$ & $B$ & $C$ & $A$ & $B$ & $C$ & $\eta \mu$ & $\eta \tau$ & $Z_{D}$ & $Z_{K}$ \\
\hline$y_{t}$ & $-2.67(1)$ & $-2.07(1)$ & $2.87(1)$ & $-2.19(3)$ & $-1.99(3)$ & $4.55(3)$ & 0.576 & 0.125 & $-2.07(1)$ & 0.576 \\
\hline$\Delta y_{t}$ & $-3.28(1)$ & $-2.11(1)$ & $-2.01(1)$ & $-4.52(3)$ & $-4.24(3)$ & $-2.57(3)$ & 0.252 & 0.120 & $-2.11(1)$ & 0.252 \\
\hline$i_{t}$ & $-2.72(1)$ & $-1.80(1)$ & $1.42(1)$ & $-2.30(3)$ & $-1.31(3)$ & $1.54(3)$ & 0.525 & 0.085 & $-1.80(1)$ & 0.525 \\
\hline$\Delta \mathrm{i}_{\mathrm{t}}$ & $-3.99(1)$ & $-4.06(1)$ & $-3.75(1)$ & $-4.98(3)$ & $-5.07(3)$ & $-4.78(3)$ & 0.102 & 0.101 & $-4.06(1)$ & 0.102 \\
\hline$(1 / \mathrm{h})_{\mathrm{t}}$ & $-3.51(3)$ & $-1.02(3)$ & $1.59(3)$ & $-1.97(3)$ & $-0.23(3)$ & $1.80(3)$ & 0.507 & 0.095 & $-1.02(3)$ & 0.507 \\
\hline$\Delta(1 / \mathrm{h})_{\mathrm{t}}$ & $-1.86(2)$ & $-2.20(2)$ & $-1.27(2)$ & $-1.29(3)$ & $-1.50(3)$ & $-1.16(3)$ & 0.148 & 0.127 & $-2.20(2)$ & 0.148 \\
\hline
\end{tabular}

Notas: Las negrillas indican rechazo de la hipótesis nula a 5\% de significancia. Los valores críticos a 5\% para la prueba Dickey-Fuller Aumentada y Phillips-Perron, en una muestra de $\mathrm{T}=100$, son de -3.45 , incluyendo constante y tendencia (modelo A), -2.89 únicamente la constante (modelo B) y -1.95 sin constante y sin tendencia (modelo C), (Maddala y Kim, 1998, p. 64). Los valores entre paréntesis representan el número de rezagos utilizados en la prueba $\eta_{\mu}$ y $\eta_{\tau}$ muestra los estadísticos de la prueba KPSs, donde la hipótesis nula considera que la serie es estacionaria en nivel o alrededor de una tendencia determinística, respectivamente. Los valores críticos a $5 \%$ en ambas pruebas son de 0.463 y 0.146 , respectivamente (Kwiatkowski et al., 1992). La prueba conjunta ADF y KPSS señala que para $Z_{D}$ la prueba estadística marcada en negrillas indica el rechazo de la hipótesis nula y para ZK indica que se acepta la hipótesis nula. La hipótesis para la prueba ADF es $H_{o}{ }^{d}$, indica que la existencia de raíz unitaria es falsa y en $H_{l}{ }^{d}$ la existencia de raíz unitaria es verdadera. Para la prueba KPSS $H_{0}{ }^{k}$ indica que la estacionaliedad es verdadera y en $H_{l}{ }^{k}$ la existencia de raíz unitaria es falsa. Los valores críticos de la prueba Dickey-Fuller y KPSS a $5 \%$ de significancia para $Z_{D}>(-5.74,0)$ y $Z_{K}<(0,0.66)$ (Charemza y Syczewska, 1998:20).

Fuente: Lugo, 2004. 
posibilidad de realizar inferencias estadísticas equivocadas (Maddala y Kim, 1998; Charemza y Syczewska, 1998). De manera adicional, se estimó la prueba conjunta de Dickey-Fuller y KPSS para hacer más preciso nuestro análisis (Charemza y Syczewska, 1998).

Los resultados de las pruebas ADF, PP y la conjunta ADF-KPSS muestran que el producto tiene un orden de integración I(1), mientras la KPSS señala que es estacionaria, con excepción del caso en el cual se incluye una tendencia determinística. Para la inversión, el resultado es similar, es decir, tiene un orden de integración I(1), ya que las pruebas ADF, PP y ADF-KPSS lo expresan, en la prueba KPSS, al considerar una tendencia determinística en la serie en niveles, el orden de integración es I(0). El índice de dispersión urbana concluye que tiene un orden de integración $\mathrm{I}(0)$. Los resultados de las pruebas ADF con constante y tendencia, KPSS y ADF-KPSS evidencian un orden de integración I(0).

Para identificar los efectos simultáneos que se establecen entre crecimiento económico y factores propuestos se estimó un modelo de vectores autorregresivos (VAR) que incluyen producto, inversión e índice de dispersión urbana. La especificación anterior pretende capturar los determinantes esenciales de un modelo de crecimiento económico del tipo fundamental en capital (AK) sin restringir las relaciones de causalidad. Los cálculos se realizaron atendiendo al orden de integración de las series para evitar la crítica de regresión espúrea (Granger y Newbold, 1974). El vaR se especificó de acuerdo con el procedimiento de Johansen (1988), para obtener un vector de cointegración que explica la relación en el largo plazo entre producto, inversión e índice de dispersión urbana con el fin de identificar los coeficientes de largo plazo entre las variables.

Por medio del procedimiento de Johansen (1988) para cointegración, se excluyó la tendencia y, de esta forma, se evitó el problema de la crítica de regresión espúrea. Así, se procedió a estimar un modelo de VAR, cuyo orden es producto, inversión e índice de dispersión.

Cuadro 3

Pruebas de cointegración basadas en el procedimiento de Johansen

\begin{tabular}{cccccc}
\hline Valores característicos & $H_{0}: r$ & $\hat{\lambda}-\max$ & $\lambda$-max 95\% & ${ }^{\wedge}$ Traza & Traza 95\% \\
\hline 0.842447 & 0 & $\mathbf{5 5 . 4 4}$ & 22.0 & $\mathbf{8 9 . 6}$ & 34.9 \\
0.544617 & 1 & $\mathbf{2 3 . 6}$ & 15.7 & $\mathbf{3 4 . 1 6}$ & 20.0 \\
0.296779 & 2 & $\mathbf{1 0 . 5 6}$ & 9.2 & $\mathbf{1 0 . 5 6}$ & 9.2 \\
\hline
\end{tabular}

Notas: $\lambda$-max $=$ Estadístico de la raíz característica máxima. ${ }^{\wedge}$ Traza. Estadístico de la traza. Las negrillas indican el rechazo de la hipótesis nula a 5\% de significancia. Periodo 1970-2001. Número de rezagos utilizados en el VAR 1, la especificación incluye constante. Valores críticos: $\lambda$-max Johansen, 1995:214, tabla 15.1, y Traza. Mackinnon et al., 1999:571, tabla II.

Fuente: Lugo, 2004.

El resultado muestra que existen más de dos vectores de cointegración en el conjunto de las variables seleccionadas. Para simplificar el análisis, sólo se supondrá la existencia de uno.

\section{Desaarrollo}


Los coeficientes normalizados indican que el vector de cointegración puede interpretarse como un mecanismo de corrección de errores de una ecuación AK. En ese sentido, los aumentos de la inversión, dispersión urbana y nivel de dispersión se traducen en mayor ritmo de crecimiento económico, el cual tiende, en un inicio, a concentrar el conjunto de actividades económicas, y con el paso del tiempo, genera economías externas y favorece, entre otras cosas, la intensidad de capital a producto. Además, destaca que la suma de ambos coeficientes de la ecuación de cointegración es igual o mayor a uno.

El vector de cointegración queda representado como: ${ }^{10}$

$$
\begin{array}{r}
\mathrm{y}_{\mathrm{t}}=12.023+0.3829 \mathrm{i}_{\mathrm{t}}+0.7170(1 / \mathrm{h})_{\mathrm{t}} \\
(0.612) \quad(0.035)
\end{array}
$$

El vector señala y mide la importancia de la inversión y dispersión urbana, en niveles, en el crecimiento económico (externalidades estáticas). Expresa mayor peso en el coeficiente de las externalidades generadas por economías externas. La suma de ambos coeficientes es aproximadamente 1.0999 y señala la presencia de rendimientos crecientes en la economía.

Cuadro 4

\begin{tabular}{|c|c|c|c|c|}
\hline \multicolumn{2}{|c|}{ Exogeneidad débil } & \multicolumn{3}{|c|}{ Causalidad de Granger } \\
\hline $\begin{array}{l}\text { Hipótesis nula } H_{0} \\
\quad \text { ECM1 }\left(\alpha_{1}=0\right)\end{array}$ & & Hipótesis Nula $H_{0}$ & & \\
\hline $\begin{array}{l}\Delta \mathrm{y}_{\mathrm{t}} \\
\Delta \mathrm{i}_{\mathrm{t}} \\
\Delta(1 / \mathrm{h})_{\mathrm{t}}\end{array}$ & $\begin{array}{lr}\chi^{2}(11)=2.93[0.08] \\
\chi^{2}(11)=1.68[0.19] \\
\chi^{2}(11)=28.16[0.0]^{*}\end{array}$ & $\begin{array}{l}\Delta \mathrm{y}_{\mathrm{t}} \text { no causa } \Delta \mathrm{i}_{\mathrm{t}} \\
\Delta \mathrm{i}_{\mathrm{t}} \text { no causa } \Delta \mathrm{y}_{\mathrm{t}} \\
\Delta \mathrm{y}_{\mathrm{t}} \text { no causa } \Delta(1 / \mathrm{h})_{\mathrm{t}} \\
\Delta(1 / \mathrm{h})_{\mathrm{t}} \text { no causa } \Delta \mathrm{y}_{\mathrm{t}} \\
\Delta \mathrm{i}_{\mathrm{t}} \text { no causa } \Delta(1 / \mathrm{h})_{\mathrm{t}} \\
\Delta(1 / \mathrm{h})_{\mathrm{t}} \text { no causa } \Delta \mathrm{i}_{\mathrm{t}}\end{array}$ & $\begin{array}{l}\chi^{2}(1)= \\
\chi^{2}(1)= \\
\chi^{2}(1)= \\
\chi^{2}(1)= \\
\chi^{2}(1)= \\
\chi^{2}(1)=\end{array}$ & $\begin{array}{l}0.03[0.85] \\
1.80[0.17] \\
0.00[0.95] \\
5.80[0.01]^{*} \\
1.31[0.25] \\
1.33[0.24]\end{array}$ \\
\hline
\end{tabular}

VECM con producto, inversión e índice de descentralización

Nota: (*) indican el rechazo de $\mathrm{H}_{0}$ a $5 \%$ de significancia respectivamente. veCM con 1 rezagos. Periodo: 1972-2001.

Fuente: Lugo, 2004.

La prueba de exogeneidad débil señala que la única variable que rechaza $H_{0}$ es el índice de dispersión. La prueba de no causalidad de Granger expresa que hay una relación causal en el comportamiento en el corto plazo entre índice de dispersión y producto. Esto se puede interpretar como una relación bidireccional entre esas variables, en la cual, si se presenta

10 El valor entre paréntesis debajo del vector de cointegración, ecuación (6), y el modelo de corrección de errores, ecuación (7), es el error estándar. 
una desviación de la posición de equilibrio, se corrige por un ajuste de largo plazo, así como por la interacción dinámica del proceso. Se confirma estadísticamente la endogeneidad de $(1 / h)$ en el modelo.

Los resultados expuestos del vector de cointegración (6) se pueden usar como mecanismo de corrección de errores (Engle y Granger, 1987). El procedimiento de lo general a lo específico permite obtener un modelo econométrico final adecuado, que puede representar una aproximación pertinente al proceso generador de información (Spanos, 1986). El modelo final queda como:

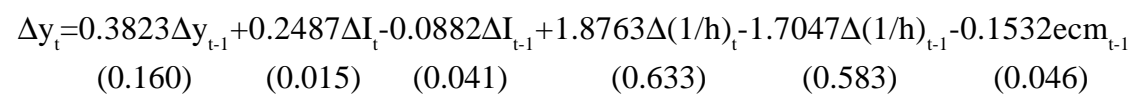
$(0.160)$
$(0.015)$
$(0.041)$
$(0.633)$
$(0.583)$

$\mathrm{R}^{2}=0.927$

RSS $=0.0028$

Autocorrelación LM(2): F = 0.4188[0.6629]

Heteroscedasticidad ARCH(2): $\mathrm{F}=1.0099[0.3786]$

Normalidad: Jarque-Bera $=1.7755[0.4115]$

Forma funcional RESET(2): $\mathrm{F}=0.3748[0.6916]$

Constancia de los parámetros:

cusum: no presenta cambio estructural.

CUSUMQ: no presenta cambio estructural.

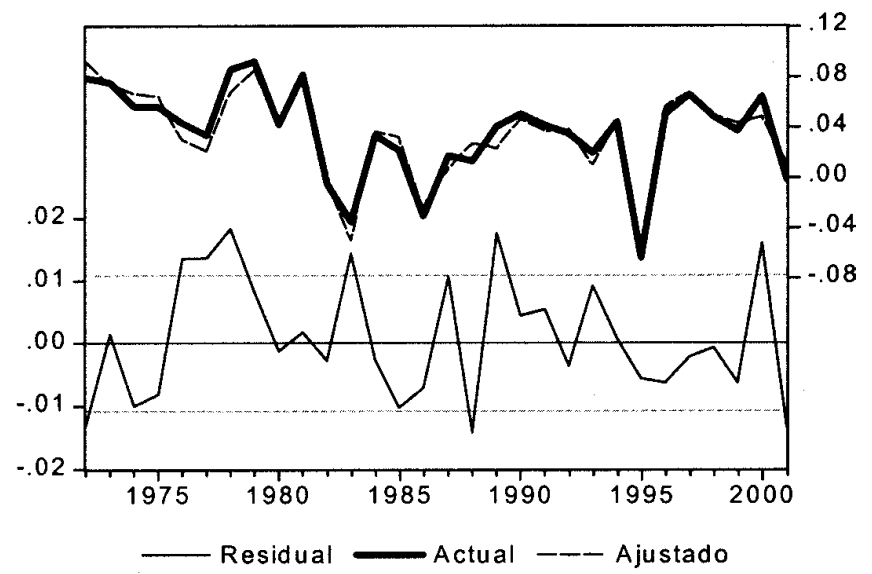

Grafica 5. Valores observados, estimados y residuales.

Fuente: elaboración propia.

Los residuales del modelo no presentan signos de autocorrelación o heteroscedasticidad. No existe problema de forma funcional y no rechaza la hipótesis de normalidad (Green, 
1999). Por lo tanto, los errores de la ecuación (7) no presentan ningún modelo sistemático que se pueda aprovechar para mejorar la estimación del modelo. Gráficamente, los coeficientes de determinación, como el comportamiento real y simulado, representan una aproximación satisfactoria de un proceso generador de información. La ecuación (7) muestra la presencia de efectos dinámicos positivos importantes de las tasas de crecimiento de la inversión y el índice de dispersión, además de un efecto de la tasa de crecimiento del producto rezagado.

\section{Conclusiones y comentarios generales}

Existe un proceso de concentración-dispersión urbana en México — periodo 1970-2001— que muestra a las externalidades como resultado del influjo de economías externas, dando como deducción un crecimiento económico nacional endógeno.

Las zonas metropolitanas seleccionadas influyen directamente en la actividad económica nacional. Generan y reestructuran sus movimientos para identificarse con economías de aglomeración que causan externalidades.

En etapas iniciales, la urbanización existe si hay un incremento en el producto, es decir, surge como parte del crecimiento económico. Acorde con un aumento en la concentración, se llega a la necesidad de pasar a una fase en la cual la saturación de zonas metropolitanas transfieren parte de sus principales actividades a regiones que al inicio de la urbanización no contaban con un nivel de desarrollo económico atractivo. Al moverse a una fase de dispersión, la relación de ésta con el producto se vuelve más estrecha y determinante, lo que genera regularidades en su relación con el crecimiento económico nacional.

Las formas estáticas y dinámicas de economías externas influyen de manera importante en el comportamiento del producto nacional. Se identifica la presencia de rendimientos crecientes que reafirma la importancia de las externalidades que en ellas se generan.

La dispersión urbana en el largo plazo es un factor explícito en el ritmo y el nivel del crecimiento económico nacional, que compensa en un periodo aproximado de 15 años la actividad y crecimiento económico de las ciudades. En el corto plazo, este factor trasciende su relación con el crecimiento del producto y nivela las bases regionales para reactivar una nueva fase de expansión de actividades y recursos económicos.

\section{Bibliografía}

Ades, A. F. y E.L. Glaeser, "Trade and Circuses: Explaining Urban Giants", Quarterly Journal of Economics, 110, 1995, pp.195-227. Agelini, E.; J. Henry, y M. Marsellino, "Interpolation with a large information set", DG- Research, working paper 72, ЕСВ and IEP-Bocconi University, IGIER Y CEPR, 2002.

Alonso, W., "Five Bell Shapes in Development", Papers of the Regional Science Association, 45, 1980, pp. 5-16.

\section{Desarrrollo}


Alperovich, G., "Economic development and population concentration", Economic Development and Cultural Change, vol. 41, núm. 1, The University of Chicago, 1992, pp. 63-74.

Arellano, J., Los esquemas metodológicos para la investigación social, México, S y G editores, 2005.

Arrow, K. J., "The economic implications of learning by doing", Review of Economic Studies, vol. 29, junio, 1962, pp. 155-173.

Asuad, N. E., Economía Regional y Urbana, México, Benemérita Universidad Autónoma de Puebla, 2001.

Barro, J. R, y X. Sala-i-Martin, Economic Growth, Mac Graw Hill, 1995.

Black, D., y J. V. Henderson, "Urban Growth”, NBER, working paper núm. 97-1, Brown University, Providence, RI, 1997; Working paper núm. 6001, Cambridge MA., April 1997.

, "A Theory of Urban Growth", Journal of Political Economy, vol. 102, núm. 2, 1999, pp. 252-284.

Bruekner, J. K., "Analyzing Third Word Urbanization: A Model with Empirical Evidence", Economic Development and Cultural Change, vol. 38, The University of Chicago, 1990, pp. 587-610.

Charemza, W. W. y D. F. Deadmean, New Directions in Econometric Practice, Edward Elgar Publishing, 1992.

Charemza, W.W. y E.M. Syczewska, "Joint application of the Dickey-Fuller and KPSs test", Econometrics Letters, vol. 61,1998, pp. 17-21.

Ciccone, A., y R.E. Hall, "Productivity and the density of economic activity", American Economic Review, vol. 86, 1996, pp. 54-70.

Clark, J. S. y J.C. Stabler, "Gibrat's law and the growth of Canadian cities", Urban Studies, vol. 28, 1991, pp. 635-639.

Combes, P., "Economic structure and local growth: France 1984-93", Journal of Urban Economics, vol. 47, 2000, pp. 329-355.

CONAPO, wWw.conapo.gob.mx

Davis, J. C. y J.V. Henderson, "Evidence on the political economy of the urbanization process", Journal of Urban Economics, vol. 53, 2003, pp. 98-125.

De Pablo, S. L., Población y planeación del desarrollo regional, México, SPP-CONAPO, 1983.

Dickey, D. A. y W.A. Fuller, "Likelihood ratio statistics for autoregressive time series with a unit root", Econometrica, 49, 1981, pp. 1057-1072.

Durlauf, S. y D. Quah., "The New Empirics of Economic Growth", NBER, Working paper 6422, Cambridge MA, 1998.

El-Shakhs, S., "Development. Primacy and Systems of cities", Journal of Development Areas, octubre 7,1972, pp. 11-36.
Engle, R. F. y C.W.J. Granger, "Cointegration and error correction: representation estimation and testing", Econometrica, vol. 55, 2,1987, pp. 251-276.

Engle, R. F. y D.F. Hendry, "Testing superexogeneity and invariance in regression models", Journal of Econometrics, vol. 56, núm. 1, 1993, pp. 119139.

-y J.F. Richard, "Exogeneity", Econometrica, vol. 51, núm. 2, 1983, pp. 119-139.

Ericsson, N.R., "Testing Exogeneity: An Introduction", en N.R. Ericsson y J.S. Irons (editores), Testing Exogeneity, Oxford University Press, N.Y., 1994, pp. 3-38.

Fay, M. y C. Opal, "Urbanization Without Growth: A not so Uncommon Phenomenon", World Bank-Country Economics Department from World Bank, Working paper 1167, 2000.

Fujita, M.; P. Krugman y A. Venables, The Special Economy, cities, regions and international trade, London, MIT Press, Cambridge, Massachusetts, 2001.

Gabaix, X., "Zipf's Law for Cities: An Explanation”, Quarterly Journal of Economics, vol. 114, 1999, pp. 739-767.

Galindo, L. M., "El concepto de exogeneidad en la econometría moderna", Investigaciones Económicas, vol. LVII:220, abril-junio, 1997, pp. 97 111

Gallup, J. L.; J.D. Sacks y A. Mellinger, "Geography and economic development", International Regional Science Review, vol. 22, 1999, pp. 179232.

Glaeser, E.L. y G. Ellison, “Geographic concentration in U. S. manufacturing industries: a dartboard approach", Journal of Political Economy, vol. 105, núm. 5, 1997, pp. 889-927.

Glaeser, E.L.; G. Dumais y G. Ellison, "Geographic Concentration as a Dynamic Process", The Review of Economics and Statistics, mayo, v. LXXXIV, núm. 2, 2002, pp. 193-204.

Glaeser, E. L.; H.D. Khalil, J.A. Scheinkman y A. Shleifer, "Growth in Cities", Journal of Political Economy, v.100, núm. 6, 1992, pp. 1126-1152.

González, L., "Retos para el desarrollo urbano en México", en Financiamiento para el Desarrollo Urbano Regional en México, coordinador Sánchez, A. A., México, XI Seminario de Economía Urbana y Regional, Instituto de Investigaciones Económicas, Programa Universitario de Estudios Sobre la Ciudad, Centro Regional de Investigaciones Multidisciplinarias de la UNAM y el Colegio Mexiquense, A.C., marzo 27-29, 2001, CD.

Granger, C.W. y P. Newbold, "Spurious regressions in econometrics", Journal of Econometrics, vol. 2, 1974, pp. 111-120.

\section{Desarrrollo}


-, N. Hyung y Y. Jeon, "Spurious regressions with stationary series", Applied Economics, vol. 33, 2001, pp. 899-904.

Green, W. H., Análisis Econométrico, Madrid, Prentice Hall, 1999.

Hansen, N., "Impact of small and intermediate- sized cities on population distribution: Issues and responses", Regional Development Dialogue II, 1990, pp. 60-76.

Henderson, J.V., "Externalities and industrial development", Journal of Urban Economics, 42, 1997, pp. 449-470

_- "Marshall's Scale Economies", NBER, working papers, paper núm. 7358, Cambridge MA, 1999.

-, "The Effects of Urban Concentration on Economic Growth", NBER, working papers, paper núm. 7503, Cambridge MA, 2000.

- "The Sizes and Types of Cities", American Economic Review, vol. 64, 1974, pp. 640-656. , "The Urbanization Process and Economic Growth: The So-What Question", Journal of Economic Growth, 8, 2003a, pp. 47-71.

, Urban Development: Theory, Facts and Illusion, Oxford, Oxford University Press, 1988.

, Urbanization, economic geography, and growth, Handbook of Economic Growth, vol. 1 , Aghion y S. Durlauf(editores), North Holland mimeo, 2003b.

- y R. Becker, "Political economy of city sizes and formation", Journal of Urban Economics, 48, 2000, pp. 453-484.

— ; A. Kuncoro y M. Turner, "Industrial development of the cities", Journal of Political Economy, vol. 103, 1995, pp. 1067-1090.

; T. Lee, y Y. Joon Lee, "Scale externalities in Korea", Journal of Urban Economics, 49, 2001, pp. 479-504.

Hendry, D.F. y K. Juselius, “Explaining cointegration analysis: part I", The Energy Journal, vol. 21, núm. 1, 2000, pp. 1-42.

, "Explaining cointegration analysis: part II", The Energy Journal, vol. 22, núm. 1, 2001, pp. 75-120.

Hirschman, A. O., "The paternity of an index", American Economic Review, Sep., 1964, pp. 761-762.

INEGI, Estadísticas Históricas de México, CD, 2000.

Iracheta, A., Hacia una Planeación Urbana Crítica, México, Gernika y UAM, 1988.

_- Planeación y Desarrollo: una visión del futuro, México, Plaza y Valdés, 1997.

Jacobs, J., The Economics of Cities, Nueva York, Random House, 1969.

Jefferson, M., "The Law of Primate City", Geographical Review, 29 (April), 1939, pp. 226-232.
Johansen, S., "Statistical analysis of cointegrating vectors", Journal of Economic Dynamic and Control, vol. 12, 1988, pp. 231-254.

— y Juselius, K., "Testing structural hypothesis in a multivariate cointegration analysis of the PPP and the UIP for UK", Journal of Econometrics, vol. 53, 1992, pp. 221-244.

Johansen, S., "Likelihood Bases Inference in Cointegrated Vector Autoregressive Models", Advance text in Econometrics, Oxford University Press, 1995.

Junius, K., "Primacy and Economic Development: Bell Shaped or Parallel Growth in Cities?", Journal of Economic Development, vol, 24(1), 1999, pp. 1-22.

Kojima, R., "Introduction: population migration and urbanization in developing countries", The Developing Economics, XXXIV- 4, 1996, pp. 349-369.

Kolko, J., "Can I Get Some Service Here: Transport Cost, Cities, and the Geography of Service Industries", Harvard University mimeo (March), 1999.

Krugman, P., "Increasing returns and economic geography”, Journal of Political Economy, vol. 99, núm. 3, 1991, pp. 483-499.

Kwiatkowski, D.; P.C.B. Phillips, P. Schmidt y Y. Shin, "Testing the null hypothesis of stationarity against the alternative of a unit root", Journal of Econometrics, vol. 54, 1992, pp. 159-178.

Levhari, D., "Extensions of Arrow Learning by Doing", Review Economics Studies, vol. 33, April, 1966a, pp. 117-131.

, "Further Implications of Learning by Doing", Review Economics Studies, vol. 33 , Juanary, 1966b, pp. 31-38.

Levine R., y D. Renelt, "A sensitivity analisys of cross-country growth regression", American Economic Review, vol. 82, 1992, pp. 942-963.

Lucas, R., "On the Mechanics of Economic Development", Journal of Monetary Economics, 12, 1988, pp. 3-42.

Lugo, I., Crecimiento económico, urbanización y concentración urbana en México 1970-2001: una aproximación por series de tiempo, México, UNAM, disertación de tesis de maestría, 2004.

MacCoskey, S. y C. Kao, "A panel data investigation of the relationship between urbanization and growth", United State Naval Academy and Syracuse University, 1998, pp. 1-24.

Mackinnon, J.G.; A.A. Haug y L. Michelis, "Numerical distribution of likelihood ratio tests for cointegration", Journal of Applied Econometrics, 14, 1999, pp. 563-577.

Maddala, G. S., Introduction to Econometrics, U. S. A., Macmillan Publishing Company, 1988. 
- y I. Kim, Unit root, cointegration and structural change, Cambridge University Press, 1998.

Mankiw, N.G.; D. Romer y D.N. Weil, "A contribution to the empirics of the economic growth", Quarterly Journal of Economics, vol. 107, núm. 2, 1992, pp. 407-437.

Marshall, A., Principles of Economics, London, MacMillan \& Co., 1890.

Mera, K., "On the Urban Agglomeration and Economic Efficiency", Economic Development and Cultural Change, vol. 21, The University of Chicago, 1973, pp. 309-324.

Mills, E. y B. Hamilton, Urban Economics, ScottForesman, 1994.

Mutlu, S., Urban Concentration and Primacy Revisted: An Analysis and some Policy Conclusions, Economic Development and Cultural Change, vol. 37, The University of Chicago, 1989, pp. 611-639.

$\mathrm{Ng}$, S. y P. Perron, "Unit root test in ARIMA models with data depend methods for the selection of the truncation lag", Journal of the American Statistical Association, vol. 90, 1995, pp. 268-281.

Ocampo, L.E., La población y el desarrollo regional y urbano, México, CONAPO, introducción, memorias del seminario, 1983.

Olivera, L.G., "Transformación metropolitana en México: efectos económicos-territoriales del comercio exterior", Comercio Exterior, vol. 47, núm. 4, 1997.

Pearce, D., The мIт Dictionary of Modern Economics, Cambridge, MA, Fourth Edition, 1992.

Phillips, P.C.B., "Time series regression with unit roots", Econometrica, vol. 55, 1987, pp. 277-301.

y P. Perron, "Testing for unit roots in time series regression", Biometrika, vol. 75, 1988, pp. 335-346.

y Xiao, Z., "A primer on unit root testing", Cowles Foundation, paper núm. 972, Yale University, 1999.

Poder Ejecutivo-SEdesol, Programa Nacional de Desarrollo Urbano 1995-2000, México, 1995.

Rauch, J., "Does history matter when it only matters a little", Quarterly Journal of Economic, vol. 108, 1993, pp. 813-867.

Romer, D., Macroeconomía Avanzada, Madrid, España, Mac Graw Hill, 2002.

___- "Increasing Returns and Long-Run Growth", Journal of Political Economy, octubre, vol. 94, núm. 5, 1986, pp. 1002-1037.

Rosen, K. y M. Resnick, "The sizes distribution of cities: an examination of the Pareto Law and primacy", Journal of Urban Economics, vol. 81, 1980, pp. 165-186.
Rosenthal, S.S. y W.C. Strange, "Evidence on the Nature and Source of Agglomeration Economies", Handbook of Urban and Regional Economics, Vol. 4., North Holland, 2003.

Ross, J., Development theory and the economics of growth, Michigan University Press, 2000.

Sala-i-Martin, X., Apuntes de crecimiento económico, Barcelona, España, Antoni Bosch Editor,1994.

Sánchez, A. A. (coordinador), Financiamiento para el Desarrollo Urbano Regional en México, México, XI Seminario de Economía Urbana y Regional, Instituto de Investigaciones Económicas, Programa Universitario de Estudios Sobre la Ciudad, CRIM-UNAM y el Colegio Mexiquense, A.C., marzo 27-29, 2001, CD.

SEDesol-CICM, Programa de 100 ciudades: una estrategia de desarrollo urbano regional sustentable y concertado, México, 1994.

— México 2020, Un enfoque territorial del desarrollo, vertiente urbana, síntesis ejecutiva, México, mayo,2000.

-, Programa Nacional de Desarrollo Urbano 1990-1994, México, 1990.

Sheshinski, E., "Optimal Accumulation with Learning by Doing", In Essays on the Theory of Optimal Growth, Cambridge, MA, edited by Karl Shell, MIT Press, 1967.

Spanos, A., Statistical Foundations of Econometric Modelling, Cambridge University Press, 1986.

Sutton, J., "Gibrat's legacy", Journal of Economic Literature, vol. XXXV, 1997, pp. 40-59.

Suzanne McCoskey y Chilhawa Kao, "A Panel Data Investigation of the Relationship Between Urbanization and Growth", wUSTL, Economics Working Paper Archive at núm. 9805, 1998.

Unikel, L., "Urbanización y Urbanismo: situación y perspectivas", en Disyuntivas sociales, presentes y futuras de la sociedad mexicana, México, colección sep/setentas, núm. 5, 1972.

Unikel, L., El desarrollo urbano de México, Diagnóstico o implicaciones futuras., México, El Colegio de México, 1976.

Wheaton, W.C. y H. Shishido, "Urban Concentration, Agglomeration Economics and The Level of Economic Development", Economic Development and Cultural Change, vol. 30, The University of Chicago, 1981, pp. 17-30.

Williamson, J., "Regional inequality and the process of national development", Economic Development and Cultural Change, June, The University of Chicago, 1965, pp. 3-45.

World Bank, Entering the 21st Century World Development Report 1999/2000, Oxford University Press, 2000.

\section{Desarrrollo}

